\title{
Valoración participativa de servicios ecosistémicos en Laguna de Nuxco, Guerrero
}

\author{
Sandy A. Medina-Valdivia, Carmen Maganda-Ramírez, \\ R. Carlos Almazán-Núñez, América L. Rodríguez-Herrera, \\ Columba Rodríguez-Alviso, José Luis Rosas-Acevedo
}

\begin{abstract}
Resumen: ¿Cómo las sociedades valoran e interactúan con los servicios ecosistémicos (SE) que les rodean para favorecer su conservación a largo plazo? Bajo la premisa que la valoración sociocultural de SE ofrece una perspectiva ampliada para potenciales contribuciones sociedad-naturaleza, presentamos aquí los resultados empíricos de una valoración participativa de SE del humedal costero Laguna de Nuxco en 2019. El diseño metodológico incluyó la combinación de herramientas cualitativas aplicadas en sitio y cuantitativas para el análisis de información. Los resultados enfatizan que los SE del humedal sustentan necesidades socioeconómicas e importantes aspectos socioculturales como patrimonio cultural e identidad como comunidades pesqueras. Identificamos conflictos socioecológicos emergentes que promueven la disminución de SE. Sugerimos adecuaciones conceptuales y metodológicas para la valoración participativa de SE en la escala local.
\end{abstract}

Palabras clave: comunidades guerrero, herramientas participativas, humedales costeros, percepción sociocultural, socioecosistemas

Las estimaciones del valor de la naturaleza en términos económicos han sido particularmente útiles para justificar la conservación de los ecosistemas. Sin embargo, la investigación contemporánea en servicios ecosistémicos (SE) ha demostrado que los enfoques monetarios son menos capaces de representar valores socioculturales de los SE (Arias-Arévalo et al., 2017; Martín-López et al., 2014), es decir, la importancia que los individuos y sus grupos sociales tiene hacia la naturaleza. Dichos valores son relevantes porque permiten entender el vínculo naturaleza-comunidades y hacer más efectivos los esfuerzos sobre la conservación de la naturaleza y sus SE. Este artículo aborda los valores socioculturales del humedal costero Laguna de Nuxco en Guerrero, México. Bajo la premisa que una 
valoración sociocultural de los SE ofrecerá una perspectiva ampliada de la relación sociedad-naturaleza para entender que hay detrás del deterioro de este humedal y tener herramientas para prevenir o corregir los agentes de cambio.

Los humedales son ecosistemas que tienen la capacidad de regular procesos ecológicos esenciales para la vida, tales como el ciclo hidrológico; de ahí que Anthony Turton los señale como "los riñones del planeta" (Turton, 2020). Asimismo, son importantes para la subsistencia y bienestar de los seres humanos ya que proporcionan una amplia gama de SE de interés ecológico, social y económico, relacionados con la calidad y disponibilidad de agua, seguridad alimentaria, biodiversidad y secuestro de carbono. México todavía enfrenta diversos retos en la gestión ambiental integrada y eficiente de los humedales, entre los que destacamos la ausencia de un escenario normativo aplicable a las actividades productivas y conservación de los humedales (Vidal et al., 2015), la insuficiente consideración de los aspectos sociales en los decretos de protección ambiental y el aparente desconocimiento de los beneficios que proveen. Esto ha propiciado la alteración de la estructura y el funcionamiento de los humedales costeros (Marín-Muñiz et al., 2016).

El sur del país refleja este escenario. En particular, el estado de Guerrero perdió más del $50 \%$ de superficie de manglares para el periodo comprendido de 1981 a 2015 debido al incremento de superficie destinada a actividades agropecuarias, acuacultura, apertura de caminos, zonas turísticas, entre otros (Vences-Martínez et al., 2016). Esta tendencia al deterioro de los humedales costeros por actividades antropogénicas señala la necesidad de prevenir o corregir los agentes de cambio que lo ocasionan (Valderrama, et al., 2017).

Nuestra investigación se enfoca en la problemática socioecológica del humedal costero Laguna de Nuxco y las localidades aledañas Colonia Veinte de Noviembre y Nuxco, que forman parte del ejido de Nuxco, situado en el municipio de Tecpan de Galeana. Estas localidades poseen un alto índice de marginación (CONAPO, 2015) ya que carecen de oportunidades sociales y servicios fundamentales para el bienestar humano, mientras basan gran parte de sus fuentes de ingresos en los SE que provee la laguna, como la pesca a pequeña escala. Por ello es relevante y necesario comprender la relación entre actores locales y el humedal frente al conjunto de beneficios que obtienen de este ecosistema costero.

El desarrollo de ganadería, agricultura y acuicultura en las zonas ejidales aledañas a la Laguna de Nuxco, generan impactos tales como cambios de uso de suelo de humedales a pastizales, deforestación y la modificación del hidroperiodo natural por la apertura artificial de la barra. Impactos responsables del deterioro de este ecosistema, reflejado en el aumento de 
sedimentos (azolve) y salinidad de la laguna, así como la degradación y disminución del 50\% de la cobertura de manglar (Vences-Martínez et al., 2016). Estas modificaciones por las actividades humanas disminuyen los SE del humedal mediante la disrupción de procesos biogeoquímicos, hidrológicos, sedimentológicos; y de las funciones ecológicas relacionadas con el control de erosión, estabilización costera y hábitat para numerosas especies de plantas y animales. Este panorama sugiere una interacción no sustentable entre las actividades socioeconómicas y la conservación de la Laguna de Nuxco, en gran parte debido a la limitada regulación para un manejo integrado que proteja al humedal y sus SE a largo plazo. En consecuencia, formulamos la siguiente pregunta de investigación: ¿cómo las comunidades interactúan y valoran los SE que les rodean para favorecer su conservación a largo plazo?

Evaluamos de manera participativa los SE de este humedal costero, de acuerdo con las prioridades socioeconómicas y valores socioculturales de los usuarios de las comunidades de Colonia Veinte de Noviembre y Nuxco. Ambas localidades utilizan el humedal, sin embargo, cada una lo hace a su manera. Los pobladores de Veinte de Noviembre basan su subsistencia en los recursos de la laguna; sus residentes hombres se dedican prioritariamente a la pesca, pero buscan alternativas de ingresos económicos cuando el recurso pesquero escasea (Medina-Valdivia, 2019). Por otro lado, la población de Nuxco hace un uso principalmente recreativo de la laguna; aunque en ocasiones ejercen la pesca, sus principales ingresos económicos derivan de otro tipo de actividades como agricultura, ganadería o jornales.

La premisa de la importancia relativa del humedal costero Laguna de Nuxco como provisor de SE para los actores locales, dependerá de sus prioridades socioeconómicas y el valor sociocultural que le otorguen. Consideramos también, en la medida que los actores locales perciban que un SE tiene la capacidad de satisfacer sus necesidades, asignarán mayor valor a este servicio, aumentando por ende su valor social.

\section{Los servicios ecosistémicos: enfoque sociocultural y participativo}

Investigaciones recientes señalan que los SE pueden servir de elemento en la toma de decisiones sobre los ecosistemas costeros ya que permiten integrar y equilibrar los diversos valores de la naturaleza (Hernández-Félix et al., 2017; Marín-Muñiz et al., 2016; Souza-Queiroz et al., 2017). Así, es posible contribuir a los desafíos de la sustentabilidad en los sistemas socioecológicos (SSE) y que, con la inclusión de las partes interesadas, apunta a 
un impacto académico y social (Jacobs et al., 2016). Este argumento ampara la relevancia de este estudio al generar evidencia de la importancia del humedal costero Laguna de Nuxco como provisor de servicios vitales para el bienestar social de las comunidades locales y brindar un elemento de peso con la apertura participativa en la toma de decisiones local para la conservación y el manejo sustentable de este ecosistema.

Utilizamos el lente de los SSE porque considera a las sociedades y a los ecosistemas como subsistemas interdependientes, que interactúan de manera compleja entre sus componentes, por lo que permite entender las dinámicas relacionadas entre sí (Maass, 2017). Esta perspectiva resulta fundamental en temas de conservación y desarrollo sustentable, porque al reconocer explícitamente los intereses, necesidades y capacidades participativas de los actores sociales, podemos integrarlos en acciones concretas a favor de la conservación. Nuestro SSE está formado por el ecosistema costero Laguna de Nuxco y las localidades Colonia Veinte de Noviembre y Nuxco.

"Los servicios ecosistémicos son los beneficios que las personas obtienen de los ecosistemas" (Evaluación de Ecosistemas del Milenio, MEA por sus siglas en inglés, 2005), esta definición de SE es la que cuenta con mayor consenso en la academia y ha sido enunciada de diferentes maneras a lo largo de los años y, en general, incluye a las características, procesos y funciones de los ecosistemas que contribuyen de manera directa o indirecta a la existencia y bienestar humanos. Los tipos de SE son: de soporte o sustento, procesos ecológicos básicos como la producción primaria neta o el ciclado de nutrientes, estos representan la base para la producción del resto de SE; de aprovisionamiento, también llamados "bienes", los cuales representan los beneficios tangibles como la madera, el agua y los alimentos (MEA, 2005); de regulación, obtenidos de los procesos de los ecosistemas como polinización, almacenamiento de carbono y protección ante amenazas naturales; finalmente los servicios culturales, que dependen del contexto sociocultural, entre los que podemos encontrar a los valores espirituales y estéticos (Arias-Arévalo et al., 2017).

Los SE culturales destacan por su intangibilidad y son particularmente relevantes para las comunidades locales de diferentes partes del mundo, dado que estas mantienen una conexión intergeneracional de relaciones con el lugar y la naturaleza, que incluso van más allá de lo material, como la identidad cultural y el conocimiento ecológico/ambiental tradicional (TEK, por sus siglas en inglés, Traditional Ecological/Environmental Knowledge), entre otros (Souza-Queiroz et al., 2017).

El estudio de los SE se convierte en un campo de acción transdisciplinario relevante para el diseño de instrumentos de gestión y política ambiental, a través de su valoración, este proceso se refiere particularmente 
a la estimación o apreciación del valor de los SE (Jacobs et al., 2016) y su desarrollo teórico y metodológico ha estado dominado principalmente por el enfoque económico (Lara-Pulido et al., 2018). Si bien estas estimaciones han sido útiles en términos de política pública, como los Pagos por Servicios Ambientales, al entregar una justificación económica para la conservación, son insuficientes para capturar todos los valores de los SE (Arias-Arévalo et al., 2017; Martín-López et al., 2014). En respuesta a esta deficiencia del enfoque económico, emergen escuelas que buscan combinar herramientas de valoración que incluyan la dimensión económica, ecológica, sociocultural y política (Jacobs et al., 2016; Perevochtchikova et al., 2019). Una de estas, es la valoración sociocultural, la cual se distingue por la inclusión efectiva de las partes interesadas para la coproducción de conocimiento y aumentar la relevancia social de los resultados (Jacobs et al., 2016).

De manera particular, el enfoque sociocultural que caracteriza esta investigación ha ganado más atención en la agenda de investigación de los $\mathrm{SE}$, situación reflejada en un número creciente de estudios que promueven la perspectiva sociocultural para valorar los SE (Boeraeve et al., 2018; Burdon et al., 2019; Scholte et al., 2015; Souza-Queiroz et al., 2017). Las diferentes experiencias basadas en el método participativo de la valoración de SE destacan sus ventajas, tales como, promover la integración de diferentes dimensiones de valor (ecológica, económica y social) para informar los procesos de toma de decisiones; fomentar el comportamiento proambiental (Rey-Valette et al., 2017); generar un aprendizaje social y responder a la demanda de mejorar el diálogo con las comunidades locales y partes interesadas para la planificación, gestión y desarrollo sostenible (Burdon et al., 2019).

Desarrollamos una valoración sociocultural participativa que considera todos los valores o importancia que los grupos o individuos asignan a los ecosistemas y sus SE (Díaz et al., 2018; Scholte et al., 2015), esto permite una visión más amplia de la contribución de la naturaleza a la cultura de las comunidades locales (Díaz et al., 2018) y los vínculos e importancia que estas otorgan a su entorno natural.

Los valores que las personas otorgan a los ecosistemas son críticos para la gestión sostenible de los SSE (Arias-Arévalo et al., 2017), estos pueden ser intrínsecos: el valor que tiene la naturaleza independientemente de su utilidad para los humanos; instrumentales: el valor de la naturaleza como medio para un fin, como el uso directo de alimentos y especies maderables (Arias-Arévalo et al., 2017); y relacionales: asociados con las relaciones y responsabilidades significativas entre los humanos y la naturaleza (Chan et al., 2016).

Nuestro estudio aboga por la consideración del valor sociocultural de la Laguna de Nuxco a través de las valoraciones de los actores locales 
sobre los SE de este humedal costero. Esto permitirá determinar los SE más relevantes en la escala local e identificar, a través de las perspectivas de los usuarios, si la distribución de estos SE es equitativa o se encuentra en espacios de confrontación y descontento por la reducción de la cantidad o calidad de los beneficios (Langholz et al., 2013). Finalmente, esto permitirá orientar los procesos de toma de decisiones para la sustentabilidad de los SSE.

\section{Protocolos y métodos del trabajo empírico}

Existen diferentes métodos para abordar la valoración sociocultural, pueden ser narrativos, deliberativos y basados en las percepciones de las partes interesadas (Rey-Valette et al., 2017), todos de carácter participativo. Para nuestro estudio, realizamos una cuidadosa selección de diferentes instrumentos metodológicos complementarios (tabla 1), esto nos permitió tener una visión amplia e integral del problema, además de capturar todos los valores de carácter sociocultural del humedal costero.

Posterior a la solicitud del Consentimiento Previo Libre e Informado, presentamos el proyecto en la localidad Colonia Veinte de Noviembre y establecimos diálogo abierto con las autoridades locales como el comisario ejidal de Nuxco; la comisaría municipal, e integrantes de la Asociación Civil "Dios, Manglar y Hombre" formada por hombres y mujeres de Colonia Veinte de Noviembre, todos, actores relevantes en la toma de decisiones.

Utilizamos un muestreo intencionado, cuyos criterios de elegibilidad fueron: (1) quiénes hacen uso directo del humedal; y (2) quiénes pueden afectar o ser afectados si existiera algún problema con el humedal y podrían brindar más información sobre los SE de Laguna de Nuxco. Así, identificamos a hombres y mujeres usuarios del humedal, habitantes de las comunidades de Colonia Veinte de Noviembre y Nuxco.

La mayoría de los participantes de este estudio son pescadores pero también trabajan en las huertas de mango y cocotero como jornaleros o peones; es decir, no poseen tierras sino que subsisten con el pago por día de trabajo. Por otro lado, las mujeres se dedican al trabajo doméstico sin retribución económica. Aunque algunas de ellas se dedican a la pesca, la mayoría se involucra en el transporte y venta de productos pesqueros en las diferentes localidades de la región Costa Grande (Medina-Valdivia, 2019). Los habitantes de Nuxco que participaron en este estudio se dedican casi de manera exclusiva a la pesca y en ocasiones, forman parte del consenso en la toma de decisiones.

Es importante señalar que, utilizamos la noción de "usuarios" para referirnos a todas las personas que participaron en este estudio, quiénes 
Tabla 1 - Diseño de la metodología para la evaluación participativa de los SE del humedal costero Laguna de Nuxco.

\begin{tabular}{|c|c|c|c|c|}
\hline & Temas & $\begin{array}{c}\text { Preguntas } \\
\text { correspondientes a los } \\
\text { temas }\end{array}$ & Instrumento & $\begin{array}{l}\text { Metodología mixta/ } \\
\text { Instrumentos }\end{array}$ \\
\hline 1 & $\begin{array}{l}\text { Conocimiento } \\
\text { del ecosistema } \\
\text { y uso de los SE }\end{array}$ & $\begin{array}{l}\text { ¿Los usuarios están } \\
\text { familiarizados con el } \\
\text { humedal? ¿Cuáles son } \\
\text { los SE que utilizan y } \\
\text { en qué o cómo son } \\
\text { utilizados? }\end{array}$ & i, iii & \multirow{7}{*}{$\begin{array}{l}\text { (i) Observación } \\
\text { participante } \\
\text { (ii) Historia de } \\
\text { vida } \\
\text { (iii) Encuesta } \\
\text { (iv) Listado libre } \\
\text { (v) Taller } \\
\text { participativo }\end{array}$} \\
\hline 2 & $\begin{array}{l}\text { Reconocimiento } \\
\text { de los SE }\end{array}$ & $\begin{array}{l}\text { ¿Cuáles son los SE que } \\
\text { reconocen los usuarios? }\end{array}$ & iii, iv & \\
\hline 3 & $\begin{array}{l}\text { Estrategias de } \\
\text { conservación } \\
\text { de los SE }\end{array}$ & $\begin{array}{l}\text { ¿Cómo cuidan/ } \\
\text { conservan los SE del } \\
\text { humedal? }\end{array}$ & iii, v & \\
\hline 4 & $\begin{array}{l}\text { Cambios } \\
\text { generales en el } \\
\text { ecosistema y } \\
\text { sus SE }\end{array}$ & $\begin{array}{l}\text { ¿Los usuarios perciben } \\
\text { cambios en los SE? } \\
\text { ¿Cómo han cambiado? }\end{array}$ & ii, iii & \\
\hline 5 & $\begin{array}{l}\text { Amenazas al } \\
\text { ecosistema y } \\
\text { sus SE }\end{array}$ & $\begin{array}{l}\text { ¿Los usuarios perciben } \\
\text { amenazas hacia el } \\
\text { humedal? }\end{array}$ & iii, v & \\
\hline 6 & $\begin{array}{l}\text { Toma de } \\
\text { decisiones y } \\
\text { Conflictos }\end{array}$ & $\begin{array}{l}\text { ¿Cómo se vinculan los } \\
\text { SE con la toma de } \\
\text { decisiones actualmente? } \\
\text { ¿Quiénes y cómo toman } \\
\text { las decisiones en torno } \\
\text { a los SE de la región? } \\
\text { ¿Existen tensiones/ } \\
\text { conflictos en torno a los } \\
\text { SE del humedal? }\end{array}$ & iii, v & \\
\hline 7 & $\begin{array}{l}\text { Compromiso } \\
\text { de conservación } \\
\text { de los SE }\end{array}$ & $\begin{array}{l}\text { ¿Los usuarios están } \\
\text { dispuestos a conservar } \\
\text { los SE del humedal? ¿Por } \\
\text { qué razones o bajo qué } \\
\text { premisas? }\end{array}$ & iii, v & \\
\hline
\end{tabular}

Fuente: elaboración propia.

forman parte de los actores locales. Sin embargo, asumimos que no logramos representar a todos los actores locales envueltos en el uso, manejo y toma de decisiones del humedal.

El paquete metodológico incluyó la observación participante durante los meses de abril a junio de 2019, cuando registramos información sobre los SE que los usuarios no mencionaron en otros instrumentos. Aplicamos 
historias de vida $(\mathrm{n}=3)$ a participantes mayores de 60 años, primeros pobladores de la localidad en condición de salud y dispuestos a establecer un diálogo fluido con el entrevistador. Estas fueron guiadas por preguntas enfocadas al tema de los cambios históricos en los SE.

Posteriormente, aplicamos una encuesta dirigida a hombres $(n=59)$ y mujeres $(\mathrm{n}=22)$ usuarios del humedal de las localidades Colonia Veinte de Noviembre $(n=75)$ y Nuxco $(n=6)$. Dicha encuesta contuvo cuatro secciones diferentes, enlazadas con los temas señalados en la tabla 1. Adicionalmente, integramos un listado libre, técnica utilizada para revisar cuando un tema es relevante o significativo para una población y qué elementos de ese tema, resaltan más, organizando los elementos mencionados. Para ello, pedimos a los entrevistados: "Mencione todos los beneficios que considere que obtiene o que le brindan el manglar y la laguna"; los beneficios se anotaron en el orden mencionado. Distinguimos esta lista como "registro de beneficios".

Cerramos con una invitación a todos los participantes de la encuesta y miembros de la comunidad Colonia Veinte de Noviembre a formar parte del taller participativo, el cual se desarrolló con los usuarios que tuvieron disponibilidad para asistir $(\mathrm{n}=20)$. La dinámica del taller consistió en hacer una mesa redonda, donde repetimos las preguntas de la encuesta a fin de propiciar el debate entre los participantes, esto permitió comparar y reflexionar sobre las respuestas obtenidas a través de otros instrumentos. Para la valoración utilizamos primero la técnica de listado libre; los participantes elaboraron una lista de todos los beneficios del humedal y posteriormente asignaron un valor a cada uno de los beneficios de la lista. Utilizamos 100 fichas para que los participantes las distribuyeran entre todos los beneficios, e hicimos énfasis en que un valor asignado a un SE le quitaría valor a otro. Así, cada uno de los beneficios de la lista obtuvo cierta cantidad de fichas, resaltando que los más importantes obtuvieron mayor cantidad. Esta actividad llevó un proceso de discusión entre los participantes para finalmente tomar un consenso de cuáles son los beneficios más relevantes para la comunidad.

El taller fue participativo porque fue un instrumento previamente planteado y consensuado con los participantes, algunos de los cuales hicieron sugerencias previas a su realización, tales como la hora y fecha del taller. Además, los participantes tuvieron un papel activo opinando y discutiendo sus diferentes experiencias y puntos de vista con respecto al uso, manejo y conservación del humedal, entre otros. Esta herramienta participativa, permite el desarrollo de capacidades para la resolución de problemas y toma de decisiones en contextos de conservación, a través de la consulta y el involucramiento de comunidades locales (Villaseñor et al., 2020). Talleres así han sido utilizados en el campo de la valoración de los SE 
porque proporciona nuevas perspectivas, conocimientos y valores que probablemente no están disponibles en la literatura científica, además de que permite identificar SE prioritarios in situ, gracias a la información suministrada por los actores locales (Scholte et al., 2015; Rey-Valette et al., 2017).

La encuesta y el taller participativo se desarrollaron del 19 al 26 de junio de 2019, fechas que estuvieron sujetas a la disponibilidad de las personas. Para el análisis de los datos obtenidos a través de los diferentes instrumentos metodológicos utilizamos el programa ATLAS.ti v.8.

Cabe señalar que, la aplicación piloto de la encuesta nos permitió adecuar los términos técnico-científicos que causaron confusión en los participantes por palabras entendibles en el lenguaje local. Así, reemplazamos el término "servicios ecosistémicos" por "beneficios", y después tradujimos el "registro de beneficios" obtenido en el listado libre a los términos formales de la investigación en SE con la clasificación de De Groot et al. (2018), un amplio conjunto de SE que muestra la mayoría de los beneficios del humedal señalados por los usuarios.

\section{Evaluación participativa de la Laguna de Nuxco}

A continuación, presentamos los resultados de la evaluación participativa de acuerdo con el orden de los componentes temáticos utilizados en la metodología.

\section{Conocimiento del ecosistema, uso y reconocimiento de los SE}

La mayoría de los usuarios entrevistados poseen un amplio conocimiento local de las especies de flora y fauna presentes en el humedal. Por ejemplo, reconocen a las especies de mangle por nombres locales, mangle bobo (Laguncularia racemosa), candelilla (Rhizophora mangle), salado (Avicennia germinans) y botoncillo (Conocarpus erectus). También, identificaron 37 especies de fauna silvestre, que incluyen aves, mamíferos, reptiles, peces e invertebrados. Estas poseen un nombre local, como pájaro buzo (Phalacrocorax brasilianus), garza morena (Ardea herodias), burrita (Fulica americana), zarzeta (Spatula discors), alcatraz (Sula nebouxii), gallinita (Porphyrio martinicus), pico espátula (Platalea ajaja), pijije (Dendrocygna autumnalis), tintin (Himantopus mexicanus) y lagarto (Crocodylus acutus).

Los usos del humedal son múltiples y no se limitan a uno solo, estos son, pesca (94\%), extracción de madera y/o fibras (33\%) para hacer aditamentos locales como ramadas, morillos, puntales, hilos, cuerda, entre otros; descanso y/o recreación (19\%), extracción de leña (7\%), extracción de sal $(5 \%)$, extracción de agua ( $4 \%)$ y uso del manglar como sombra $(2 \%)$. 
Por otro lado, el $100 \%$ de los usuarios reconocen que el humedal costero les brinda beneficios de manera individual y de manera colectiva. Tal como lo indica el siguiente testimonio:

“De la laguna sacamos todo para la canasta básica, y el manglar nos sirve para que la laguna tenga agua ¡Si no hay manglar, la laguna se secaría! yo siempre lo he visto como un paraíso el que tenemos aquí, un privilegio de Dios" (pescador y técnico, 54 años).

En la sección del listado libre, elaboramos el "registro de beneficios" sin modificar las palabras o frases utilizadas por los usuarios. Estos elementos se ordenaron de mayor a menor frecuencia de citas (e.g., "pesca" 41 citas; "pescado" 24 citas; sombra" 16 citas, etc.) (figura 1).

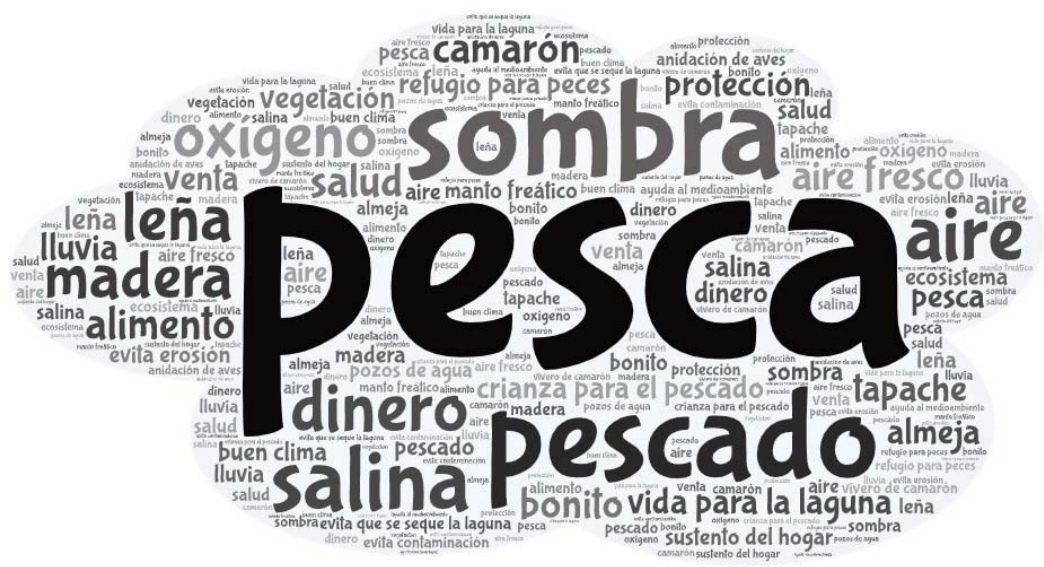

Figura 1 - Principales beneficios percibidos por los usuarios del humedal costero Laguna de Nuxco. Las palabras o frases mencionadas con mayor frecuencia se distinguen por un tamaño de letra mayor. Fuente: Encuesta aplicada a usuarios.

\section{SE relevantes para los usuarios locales}

A partir del "registro de beneficios", evaluamos la importancia relativa de los SE a través de dos indicadores, la frecuencia de citas (figura 2) y la clasificación jerárquica (ranking) de los SE identificados para evaluar (figura 3). Para el ranking, utilizamos las posiciones de los SE en el listado (Rey-Valette et al., 2017). Por ejemplo, el SE de "regulación del clima" fue colocado cuatro veces en el primer lugar de la lista y 15 veces en el segundo. Es importante señalar que, cuando algunas de las respuestas eran alusivas al mismo SE, las frecuencias de citas se sumaron, por ejemplo, "vida para la laguna" citada 11 veces y "crianza para el pescado" citada 


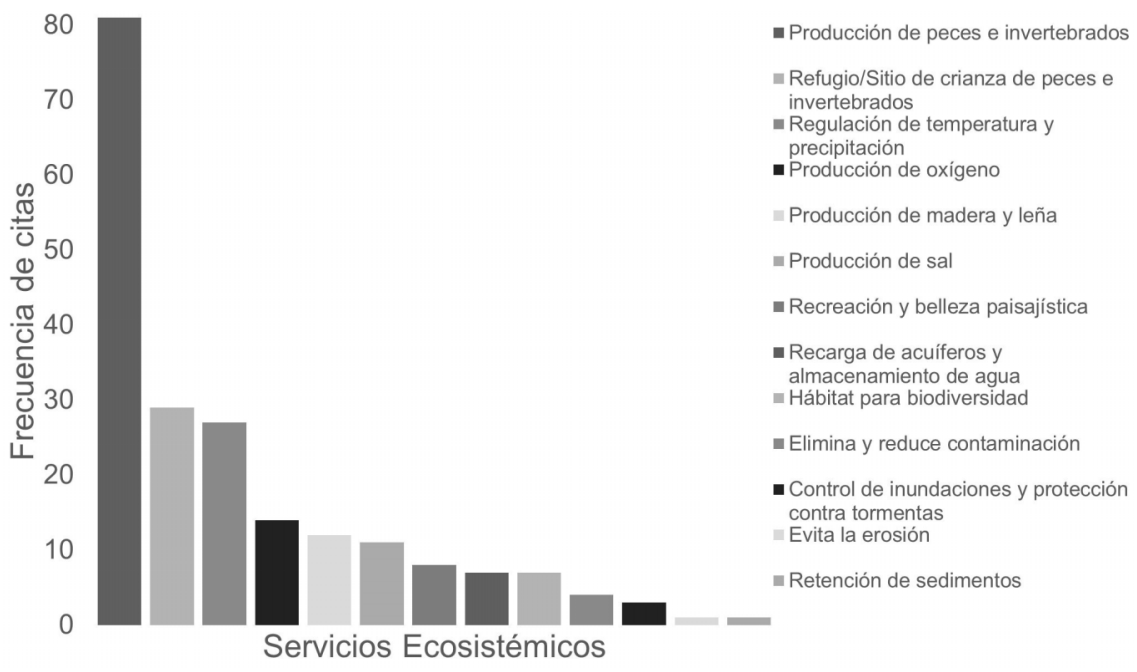

Figura 2 - Resultado indicador 1: SE del humedal costero Laguna de Nuxco relevantes para los usuarios, de acuerdo con la frecuencia de citas.

9 veces, sumaron 20 citas al SE de "refugio/sitio de crianza de peces e invertebrados".

En la encuesta, los usuarios identificaron 13 SE proporcionados por el humedal costero Laguna de Nuxco; cinco de regulación, cuatro de soporte/hábitat, tres de aprovisionamiento y uno cultural (tabla 2). El SE

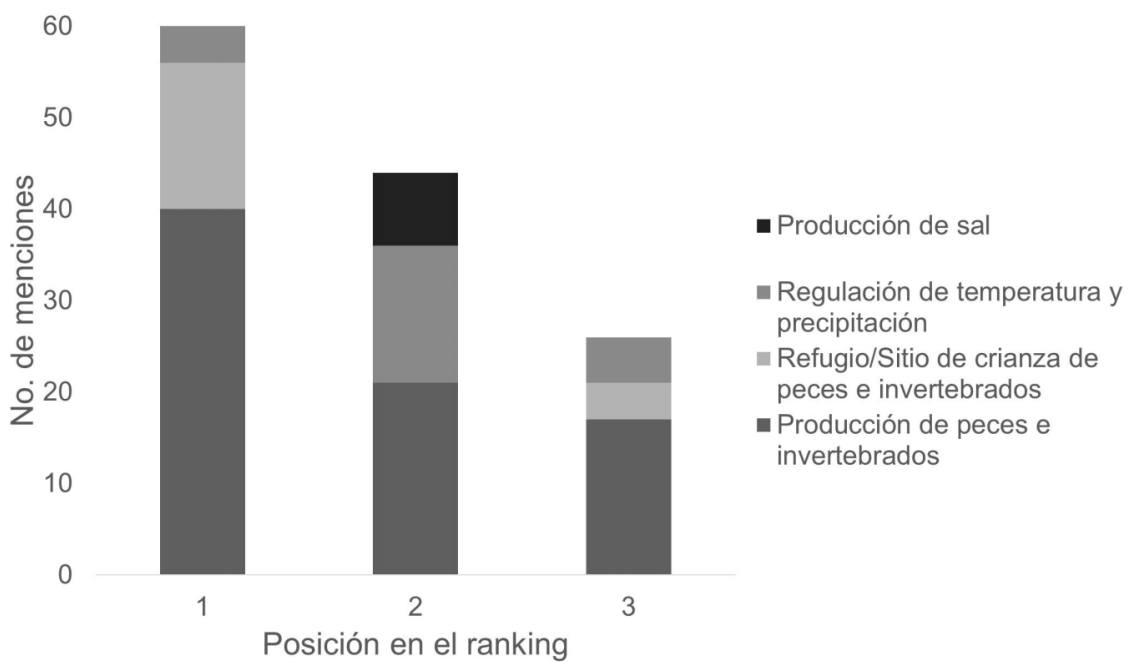

Figura 3 - Resultado indicador 2: SE del humedal costero Laguna de Nuxco relevantes para los usuarios, de acuerdo con su posición $\left(1^{\mathrm{a}}, 2^{\mathrm{a}}\right.$ y $\left.3^{\mathrm{a}}\right)$ en el ranking de servicios. 
Tabla 2 - SE del humedal costero Laguna de Nuxco percibidos por usuarios de las localidades Colonia Veinte de Noviembre y Nuxco.

\begin{tabular}{|c|c|}
\hline Categorías & Servicios Ecosistémicos \\
\hline \multirow{3}{*}{ Aprovisionamiento } & $\begin{array}{l}\text { Alimentación } \\
\quad \checkmark \text { Producción de peces e invertebrados }\end{array}$ \\
\hline & $\begin{array}{l}\text { Fibra y combustible y otras materias primas } \\
\checkmark \text { Producción de madera y leña }\end{array}$ \\
\hline & $\begin{array}{l}\text { Productos químicos } \\
\checkmark \text { Producción de sal }\end{array}$ \\
\hline \multirow{5}{*}{ Regulación } & $\begin{array}{l}\text { Regulación del clima } \\
\checkmark \text { Regula la temperatura y la precipitación }\end{array}$ \\
\hline & $\begin{array}{l}\text { Regímenes hidrológicos } \\
\checkmark \text { Recarga de aguas subterráneas y almacenamiento } \\
\text { de agua }\end{array}$ \\
\hline & $\begin{array}{l}\text { Control de la contaminación y desintoxicación } \\
\checkmark \text { Retención y eliminación del exceso de } \\
\text { contaminantes }\end{array}$ \\
\hline & $\begin{array}{l}\text { Mitigación de riesgos naturales } \\
\checkmark \text { Control de inundaciones y protección contra } \\
\text { tormentas }\end{array}$ \\
\hline & $\begin{array}{l}\text { Protección contra la erosión } \\
\checkmark \text { Retención de suelos }\end{array}$ \\
\hline \multirow{3}{*}{ Culturales } & $\begin{array}{l}\text { Patrimonio cultural e identidad } \\
\checkmark \text { Sentido de lugar y pertenencia }\end{array}$ \\
\hline & $\begin{array}{l}\text { Inspiración espiritual y artística } \\
\checkmark \text { Bienestar y felicidad } \\
\end{array}$ \\
\hline & $\begin{array}{l}\text { Actividades recreativas y estética } \\
\checkmark \text { Visitantes locales y belleza paisajística }\end{array}$ \\
\hline \multirow{4}{*}{ Soporte/Hábitat } & $\begin{array}{l}\text { Hábitat } \\
\quad \checkmark \text { Refugio/Sitio de crianza de peces e invertebrados }\end{array}$ \\
\hline & $\begin{array}{l}\text { Fotosíntesis } \\
\quad \checkmark \text { Producción de oxígeno }\end{array}$ \\
\hline & $\begin{array}{l}\text { Biodiversidad } \\
\checkmark \text { Hábitat para especies de aves residentes o } \\
\text { transitorias }\end{array}$ \\
\hline & $\begin{array}{l}\text { Formación del suelo } \\
\checkmark \text { Retención de sedimentos y acumulación de materia } \\
\text { orgánica }\end{array}$ \\
\hline
\end{tabular}

Fuente: elaboración propia a partir de los resultados de la encuesta y taller participativo. Se utilizan las categorías de SE de humedales costeros de De Groot et al. (2018). 
más valorado y citado fue el de "producción de peces e invertebrados", y estuvo posicionado el mayor número de veces en la primera posición del ranking de servicios. Seguido de este, están el servicio de soporte "refugio/sitio de crianza de peces e invertebrados"; y el servicio de regulación "regulación de temperatura y precipitación". Otros servicios notables fueron el servicio de soporte "producción de oxígeno"; y los servicios de aprovisionamiento "producción de sal" y "producción de madera y leña".

Los resultados de la actividad de valoración en el taller participativo fueron bastante similares a los de la encuesta, siendo el SE de mayor valor el de "alimentación", derivado de la producción de peces. Los usuarios también identificaron otros SE clasificados como culturales, "sentido de lugar y pertenencia" y "bienestar y felicidad".

\section{Estrategias de conservación y cambios generales de los SE}

La principal práctica que realizan los usuarios para mantener los SE del humedal costero es limpiar el humedal de RSU (residuos sólidos urbanos) y evitar la mala disposición de estos (44\%). También, evitar la tala de manglar (43\%) es una de las estrategias más importantes. Otras estrategias señaladas en menor medida son: cuidar cuando se abre la barra, no contaminar el agua y usar malla pequeña. "Afortunadamente sabemos con algunos compañeros pescadores en qué debemos cuidar el manglar y la laguna, y aquí cada uno cuando sale a pescar, anda recogiendo los botes de refresco que la gente tira. La laguna es nuestra casa también y hay que cuidarla" (pescador y jornalero, 35 años).

En el taller participativo destacó la apertura artificial de la barra y fue corroborada en las visitas piloto. Esta acción se realiza con diferentes objetivos, como evitar inundaciones en las localidades y zonas de pastoreo (potreros) y para mejorar la pesquería con la afluencia de especies de valor comercial que ingresan al humedal a través del intercambio de agua del océano y la laguna.

De acuerdo con los resultados de la encuesta, la deforestación de las áreas de manglar, la contaminación por RSU y el crecimiento de la población han sido las principales causas de cambio en el humedal costero (figura 4). Esta información fue apoyada en las historias de vida, dado que los primeros pobladores especificaron que existía mayor cobertura de manglar. Asimismo, algunos usuarios señalaron en sus testimonios la pérdida de algunas especies de invertebrados acuáticos, tal como lo indica el siguiente testimonio:

Antes había caracol grande, había una almeja blanca grande también. . . venía un señor del embarcadero, que se llevaba costalillas de caracoles, 
Don Tomás El Caracolero, las vendía en Acapulco para hacer los collares, ceniceros, todas esas cosas. Cuando se iba secando la laguna, en toda la orilla de la laguna, en vez de pisar la laguna, pisabas puro caracol. . . ya todo eso se acabó. (pescador y jornalero, 49 años)

\section{Amenazas al ecosistema, toma de decisiones y conflictos}

En los resultados de la encuesta, el $80 \%$ de los usuarios perciben que existen amenazas que pudieran afectar al humedal y la provisión de los SE. La principal, es la fumigación de las huertas de mango que rodean el humedal; como indica el testimonio:

Desde que empezaron a fumigar, se acabó la godorniz y se acabó la iguana... eso es una cosa muy grave que yo he visto que ha afectado mucho a la laguna, incluso hemos encontrado animalitos muertos, aves muertas en la laguna... ¿Y la fumigación quien la hace? Pues los mangueros (pausa) pero, yo quisiera que de eso no de nombres. (pescador, 54 años)

Otras amenazas son, la falta de lluvias y el calentamiento del agua de la laguna; la contaminación de RSU (botellas de plástico, botes de herbicidas, plaguicidas); la construcción de viveros de camarón, resaltada como una amenaza mayor debido a los impactos que produce como dragado del terreno, deforestación zonas de manglar, extracción de agua de la laguna y el vertido de agua residual contaminada por tratamientos químicos; la deforestación; la falta de cultura y responsabilidad de la población y la desaparición del mejillón (molusco bivalvo Mytella strigata). En el taller

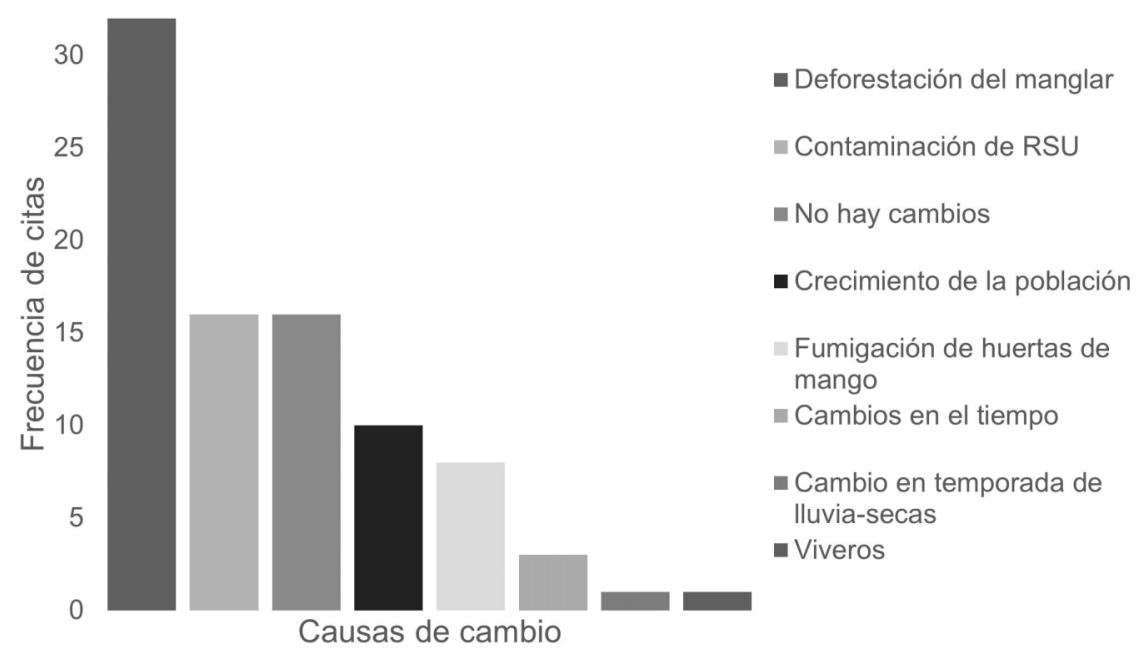

Figura 4 - Causas de cambio en el humedal costero percibidas por los usuarios. Fuente: Encuesta aplicada a usuarios. 
participativo, las aguas negras provenientes de los drenajes que son vertidos en la laguna, fue adicionada a esta lista.

Los usuarios reconocen las instancias para la organización y toma de decisiones en torno al humedal. En orden de importancia son: Comité de Barra/Laguna; Asociación Civil "Dios, manglar y hombre", comisaría ejidal; comisaría municipal; e instituciones gubernamentales como Dirección de Ecología Municipal, Secretaría de Medio Ambiente y Recursos Naturales (SEMARNAT) y Secretaría de Agricultura y Desarrollo Rural (SAGARPA). También afirmaron la existencia de consenso para la toma de decisiones ya que, aunque el Comité de Barra/Laguna tiene el liderazgo, los acuerdos de lo que sucede en el humedal se realizan en reuniones donde se convoca a toda la localidad. En ese sentido, el comité de laguna es un organismo local, surgido de la propia comunidad Colonia Veinte de Noviembre, conformado por voluntarios locales, quiénes organizan las actividades comunitarias relacionadas con el humedal, tales como vigilancia, limpieza, vedas y más. La mayoría de los usuarios (94\%) están de acuerdo con las decisiones tomadas en consenso, siempre y cuando sea para el bien del humedal. Lo que refleja unidad social ante las decisiones locales.

En cambio, identificamos tres conflictos relacionados con el humedal costero. El primero involucra a los sectores agrícola y pesquero, y está relacionado con la contaminación del humedal debido a la fumigación aérea con productos químicos para las huertas de mango aledañas al humedal. El segundo, involucra al sector privado y organización no gubernamental (ONG), así como a las instituciones Procuraduría Federal de Protección al Ambiente (PROFEPA) y SEMARNAT, a las cuales se dirigen los usuarios del humedal para denunciar la afectación de considerables áreas de manglar por la construcción de viveros de camarón. El tercer conflicto está relacionado con la apertura artificial de la barra, actividad que requiere organización entre los habitantes de la Colonia Veinte de Noviembre en conjunto con autoridades locales y otras comunidades. Este último conflicto se debe a la incompatibilidad de intereses y necesidades entre actores locales de diferentes sectores socioeconómicos, pesquero, agrícola, ganadero y población en general. Por ejemplo, algunos agricultores se oponen a abrir la barra, señalando la necesidad de mantener la humedad en el subsuelo para favorecer los cultivos; mientras que pescadores están a favor para beneficiar la pesca con el ingreso de larvas de especies de valor comercial; pero se oponen a la apertura anticipada para generar condiciones adecuadas de precipitación.

En todo caso, los usuarios están de acuerdo en que el cuidado del humedal es responsabilidad de todos por el motivo central de que su economía y bienestar personal y familiar, depende de los beneficios que 
les brinda. Asimismo, señalaron que las autoridades deberían vigilar las actividades que se realizan en el humedal o proporcionar un apoyo económico a los pobladores para reforzar las actividades de cuidado que ya realizan.

\section{Discusión}

\section{Conocimiento del ecosistema, uso y reconocimiento de los SE}

Los resultados muestran que los usuarios de las localidades Colonia Veinte de Noviembre y Nuxco poseen un valioso conocimiento local de la Laguna de Nuxco y sus medios de vida están estrechamente vinculados a los SE de este humedal costero.

Al igual que en otras comunidades asentadas cerca de ecosistemas de manglar, la pesca es un significativo medio de sustento (HernándezFélix et al., 2017). No obstante, Vences-Martínez et al. (2018) señalan que a partir de la década de los noventa, los usuarios de Laguna de Nuxco percibieron una disminución en la abundancia de especies acuáticas debido a disturbios en el manglar y el uso de técnicas de pesca indiscriminada que hicieron insostenible la actividad pesquera. Consideramos que este escenario puede mejorar si los directos interesados, los pescadores, participan activamente en la planificación y gestión del humedal para el establecimiento formal de reglas de pesca para todos los usuarios locales y foráneos, además de retomar los acuerdos locales que existen y que suelen no ser respetados por todos; por ejemplo, el uso de "luz de malla" que no captura peces juveniles.

La colecta de madera y leña es un uso tradicional en los humedales costeros, particularmente la extracción de leña en Laguna de Nuxco es uno de los usos relevantes para los usuarios. Sin embargo, existe entre ellos el conocimiento de que las especies de mangle están protegidas por leyes federales y que su extracción es ilegal; por lo que señalaron, han reducido su extracción. Frases como, "antes se podía cortar" o "está prohibido" fueron comunes entre los usuarios. A pesar de las prohibiciones gubernamentales, los usuarios recurren a lo que podríamos llamar la recolecta clandestina de leña y corte clandestino de árboles de mangle. Por lo tanto, son necesarias estrategias de manejo aterrizadas al lugar para evitar el temor entre los usuarios del humedal y se realice un aprovechamiento sustentable de la madera y la leña.

Por otra parte, los usuarios reconocen al humedal costero Laguna de Nuxco como un importante proveedor de beneficios de interés particular y comunitario. La mayoría de los SE identificados-principalmente de provisión-poseen un valor instrumental como un medio para lograr un 
fin particular (Pascual et al., 2017). Los SE más valorados por los usuarios están directamente relacionados con la provisión de peces, como el servicio de aprovisionamiento "producción de peces e invertebrados" y el servicio de soporte "refugio/sitio de crianza de peces e invertebrados". La atribución de mayor valor a estos SE sugiere la importancia del humedal costero como soporte para la alimentación y los medios de vida de las comunidades aledañas. Esto coincide con lo expuesto por Marín-Muñiz et al. (2016) en humedales de Monte Gordo, Veracruz, donde el SE de "sitio de crecimiento de peces" fue altamente percibido tanto en la población productiva, como en jóvenes estudiantes.

Otro SE relevante es el servicio de "regulación de temperatura y precipitación", ligado principalmente al bosque de mangle. Este recurso provee "sombra fresca" en las jornadas de trabajo de los pescadores y mientras que los árboles de mangle llaman el agua, es decir promueven la precipitación. De acuerdo con la MEA (2005), el mantenimiento de un clima favorable es un SE que posee una tendencia a la baja en la escala local debido a las transformaciones del territorio como los cambios de uso de suelo.

En contraste con otros estudios, como los de Marín-Muñiz et al. (2016) y Hernández-Félix et al. (2017), donde el servicio de "regulación de clima" no resalta entre sus resultados, probablemente porque los participantes no tenían contacto directo con el manglar; en nuestro estudio este servicio fue citado frecuentemente porque los participantes son en su mayoría pescadores quiénes se benefician de la sombra del manglar. Además, debido a las características de vegetación y uso de suelo de la zona de estudio, el manglar es un bosque siempre verde en medio de una matriz agropecuaria con parches de Selva Baja Caducifolia, que la mayor parte del año permanece seca.

El servicio de soporte "producción de oxígeno" también se destacó entre los demás servicios. Dado que los usuarios utilizaron específicamente "oxígeno" en las respuestas, este resultado podría ser por la intervención de estudios previos relacionados con el humedal y las localidades. El estudio de Vences-Martínez et al. (2018), quiénes interactuaron con la comunidad sobre el cuidado del manglar; más otros estudios de la Universidad Autónoma de Guerrero acerca de los niveles de oxígeno en la laguna y la estructura del manglar; así como las actividades de gestión de una ONG que han favorecido el acercamiento de autoridades ambientales como la Comisión Nacional Forestal (CONAFOR), SEMARNAT y PROFEPA, promueven el uso de estos términos técnicos entre los usuarios.

Otros dos SE notables son, el servicio de aprovisionamiento "producción de sal", que fue señalado principalmente por los usuarios que se han dedicado a la extracción de sal y que la realizan de manera artesanal y el servicio de aprovisionamiento "producción de madera y leña". Probable- 
mente, este último SE no fue resaltado de manera intencional porque entre los usuarios existe la percepción de que al extraer madera o leña del manglar serán acreedores de sanciones gubernamentales. Este resultado también está relacionado con los diferentes tipos de leña obtenida del árbol seco, la madera se obtiene al talar el árbol y esa es justamente una de las acciones que los usuarios evitan para mantener los beneficios del manglar. Sin embargo, en la observación participante nos percatamos que la leña es un combustible utilizado por la mayoría de los usuarios, lo que resalta la importancia del humedal como proveedor de materias primas.

Los SE culturales como "visitas al humedal" y "belleza paisajística" fueron los menos valorados por los usuarios encuestados, y este resultado contrasta con otros estudios similares. Souza-Queiroz et al. (2017) detallan que el servicio cultural de "recreación y turismo" fue altamente valorado por los pescadores de Cumbe en Brasil, quiénes además de la pesca realizan actividades al aire libre como la natación, paseos soleados y reuniones sociales, en un entorno altamente influenciado por el turismo tradicional donde los manglares son utilizados por prestadores de servicios turísticos locales, quiénes alternan entre pesca y turismo como actividades económicas. Mientras tanto, en Laguna de Nuxco, solo se llevan a cabo actividades recreativas como visitas de personas locales a la bocabarra principalmente los fines de semana. Otro aspecto que influyó es que los participantes de este estudio son principalmente pescadores, ellos permanecen la mayor parte del tiempo pescando en el humedal de lunes a sábado, mientras que el domingo descansan en sus casas.

Los SE culturales identificados en el taller participativo se relacionan principalmente con los modos de vida de la comunidad y con las relaciones significativas que han forjado con el humedal a lo largo de los años. Expresiones como "sentido de lugar y pertenencia", reflejaban la satisfacción de sentirse parte del grupo "pescadores o pescadoras de El Veinte" y "bienestar y felicidad" que perciben los usuarios al estar en la laguna y el manglar.

Nuestro estudio reveló que los conocimientos empíricos de pescadores y pescadoras en su constante interacción con el humedal son un servicio cultural importante en las comunidades, aunque no hayan sido referidos directamente como un "beneficio", sino como un legado que las siguientes generaciones deberían conservar. Esta información forma parte del TEK, y entre los usuarios del humedal está relacionado con la identidad sociocultural basada en la pesca. TEK es definido como el conjunto acumulado de conocimientos, prácticas y creencias locales entre los seres humanos y su entorno, transmitido de generación en generación y reconocido por promover la conservación de los ecosistemas por las comunidades locales (Pascual et al., 2017). 
Los SE culturales descubiertos en esta valoración enmarcan una dimensión relacional, que de acuerdo con Chan et al. (2016) reconoce los lazos sociales con la tierra y trasciende la dicotomía de los humanos separados de la naturaleza, promoviendo así, relaciones socioecológicas sostenibles y altamente satisfactorias para las generaciones presentes y futuras.

La principal diferencia entre los valores asignados por los usuarios a los SE radica en que, algunos beneficios que obtienen del humedal pueden ser comparados en términos económicos, por ejemplo, el costo de los productos pesqueros de los cuales obtienen dinero y satisfacen sus necesidades socioeconómicas. Por otro lado, algunos beneficios poseen un valor no monetario, como pensamientos o sentimientos en torno la satisfacción de mirar el paisaje de la laguna, formar parte de un grupo de pescadores; o heredar los conocimientos sobre el arte de pesca; los cuales satisfacen necesidades socioculturales. Dado que ambos tipos de valor son relevantes para los actores locales, deberían integrarse en la toma de decisiones a nivel comunitario.

\section{Estrategias de conservación y cambios generales en el ecosistema y los SE}

Las estrategias comunitarias para la conservación de los SE son acciones voluntarias que realizan los usuarios del humedal, sin decreto gubernamental que les obligue a llevarlas a cabo. Por lo que representan la disponibilidad de los residentes locales para cuidar y mantener los beneficios que le otorgan los sistemas naturales de manera voluntaria.

En este estudio, la actividad de limpieza de RSU del humedal es la primera estrategia que utilizan los usuarios. Dicho resultado se debe probablemente a la cantidad de basura que es arrastrada en temporada de lluvias al humedal debido al manejo ineficiente de RSU en las diferentes localidades aledañas a la laguna. Un caso similar es el estudio de Hernández-Félix et al. (2017) quiénes señalaron el "manejo de desechos" como una de las estrategias relevantes para cuidar el manglar en Punta Arena, un área aledaña a la Reserva de la Biosfera Ría Celestún.

En la segunda posición de las estrategias comunitarias se encuentra "evitar la tala de manglar". Es posible que este resultado está influenciado por experiencias previas, como la aplicación de sanciones económicas a algunos miembros de la localidad que talaron zonas de manglar. Sin embargo, la "deforestación del manglar" fue percibida como la principal causa de cambio a lo largo de los años, además de una amenaza actual para el humedal y sus SE. Cabe destacar que los usuarios no solo perciben este cambio en el ecosistema, sino que optan por prevenirlo, en este caso, evitando la tala de manglar. 
La deforestación de zonas de manglar fue percibida como la principal causa de cambio del humedal. Esta actividad ha incrementado con el tiempo, fundamento para atender los aspectos promotores del cambio señalados anteriormente, como el cambio de uso de suelo de humedales a pastizales y el desarrollo de acuacultura en las zonas aledañas al manglar (Medina-Valdivia \& Maganda-Ramírez, 2019; Vences-Martínez et al., 2016).

La pérdida de manglar puede traer consigo consecuencias socio-ecológicas importantes, como el incremento de la vulnerabilidad a inundaciones (Marín-Muñiz et al., 2016) que afectará principalmente a quiénes viven cerca del humedal. Además de la pérdida de hábitat para las especies de peces e invertebrados de las que depende la actividad pesquera local.

Resulta contradictorio que a pesar de que las acciones locales de limpieza de los RSU fue una de las más resaltadas, el problema de los RSU fue señalado como la segunda causa de cambio. Esta situación también fue expuesta por Vences-Martínez et al. (2018), quiénes registraron que el principal problema ambiental del humedal Laguna de Nuxco eran los RSU.

Ambos cambios son altamente percibidos por los usuarios quiénes señalan que los beneficios que les brinda el humedal podrían mermar como consecuencia del deterioro ecológico de este ecosistema.

\section{Amenazas al ecosistema, toma de decisiones y conflictos}

Este estudio no estuvo centrado en la identificación de conflictos, no obstante, a partir de los resultados de estos dos componentes temáticos, identificamos tres principales conflictos relacionados con el humedal. De acuerdo con la tipología de conflictos socioambientales de Langholz et al. (2013), estos conflictos se encuentran en la etapa de emergentes, es decir, los actores son conscientes de la afectación al humedal costero y expresan su descontento de manera pacífica.

Los conflictos embebidos en el SSE que analizamos surgen principalmente por la incompatibilidad de intereses y necesidades de actores económicos diversos. Rincón-Ruiz et al. (2019) señalan que los conflictos ambientales son comunes en Latinoamérica, dado que existe un alto grado de exclusión social e inequidad, además de que estos pueden dificultar la aplicación de la valoración de SE. Particularmente, la presencia de conflictos no dificultó esta valoración, pero presenciamos momentos de tensión y hostilidad verbal entre actores, algunos de los cuales, no participaron en este estudio.

Algunos usuarios señalaron la existencia de acuerdos locales para regular algunas actividades en la laguna, como el respeto a los espacios de 
pesca para cada una de las comunidades o la prohibición de lanchas de motor, no obstante, estos acuerdos son rebasados, incluso por aspectos de inseguridad social. Vences-Martínez et al. (2018) también resaltaron la necesidad de la intervención de instancias gubernamentales para solucionar los problemas del uso irracional de los recursos de este humedal, pero aún no existen soluciones aterrizadas para mejorar este escenario.

Esta investigación demuestra que la conservación del humedal es sumamente importante no solo en términos ecológicos, sino porque las comunidades locales comprenden la importancia de los ecosistemas para su propia sobrevivencia y para el mantenimiento de su identidad como comunidades pesqueras. Sin embargo, las decisiones individuales o de pequeñas asociaciones gremiales, seguirán siendo rebasadas sino existe un organismo regulador de las actividades socioeconómicas que generan mayor impacto en el humedal.

Finalmente, sugerimos ampliar los campos de investigación, por ejemplo, hacia las funciones ecológicas subyacentes del humedal costero y que están relacionadas específicamente con los SE de mayor importancia para las comunidades locales. Así como incorporar el uso de herramientas como los Sistemas de Información Geográfica que aportan mayor conocimiento a la distribución espacial de los SE y son utilizados como instrumentos prácticos en los procesos de toma de decisiones (Burdon et al., 2019).

\section{Conclusiones}

Nuestra pregunta central fue ¿cómo las comunidades locales interactúan y valoran los SE que les rodean para favorecer su conservación a largo plazo? Con el desarrollo de la investigación podemos responder que los actores sociales valoran e interactúan con el humedal costero Laguna de Nuxco de forma diferenciada, en pleno uso y reconocimiento de los $\mathrm{SE}$, conscientes de los cambios y amenazas en el ecosistema, así como generadores de potenciales estrategias de conservación y coexistencia con otras actividades económicas para evitar conflictos. Este ecosistema en particular proporciona utilidades individuales como alimentación y combustible y de desarrollo económico, como trabajo, educación y patrimonio. Además, otorga relaciones significativas entrelas comunidades locales y la naturaleza que las rodea, como patrimonio cultural e identidad como comunidades pesqueras. Estos valores económicos y socioculturales son el punto de partida para mejorar la toma de decisiones en el nivel local y construir estrategias comunitarias que permitan mantener los SE a largo plazo. 
Todos los SE que provee el humedal costero Laguna de Nuxco, promueven la calidad de vida de los habitantes de todas las comunidades aledañas. Sin embargo, los actores sociales inmersos en el SSE están familiarizados con algunos de los SE pero adolecen de otros que pueden generar estrategias de conservación de mayor alcance y no estar limitadas a la limpieza de RSU o a evitar talar el manglar.

La falta de regulación de las actividades que se desarrollan en el humedal y sus áreas adyacentes favorece la existencia de conflictos socioambientales. Estos conflictos contribuyen a la disminución y acceso desigual al suministro de SE del humedal y ponen en riesgo el bienestar de las comunidades locales.

El estado actual del humedal demanda de forma urgente, una normatividad que garantice la salud del ecosistema costero y promueva la diversificación de los medios de vida mediante actividades de bajo impacto ecológico. $\mathrm{Al}$ respecto, el humedal posee elementos ecológicos para desarrollar este tipo de actividades, por ejemplo, es un sitio prioritario para la conservación de las aves y forma parte del Área para la Conservación de las Aves (AICA) Lagunas Costeras de Guerrero. Este elevado potencial para el turismo de observación de aves, práctica a nivel mundial, es un segmento destacado del turismo ecológico comunitario. Entre los actores locales existe el interés de ampliar las actividades socioeconómicas con actividades de bajo impacto ecológico que, además de promover un pensamiento a favor del cuidado del humedal, se apuntala como una oportunidad para impulsar la economía local.

Reconocemos que los participantes de este estudio tienen frente así la disyuntiva de convertirse en actores protagónicos de un cambio participativo, para prevenir que los impactos ecológicos que enfrentan hoy se conviertan en irreversibles, afectando su bienestar, suficiencia alimentaria, salud y la estabilidad social local y regional.

La valoración participativa requiere una aproximación piloto para resolver algunos desafíos como la multiplicidad de SE, los cuales deben ser adaptados a una clasificación que permita mostrar toda la diversidad de beneficios identificados por los usuarios locales. Además, la investigación sobre SE en el nivel local debe emplear diferentes instrumentos metodológicos para recopilar y triangular la información obtenida, así como involucrar a los actores locales en el desarrollo.

Nuestros resultados contribuyen al debate teórico y metodológico de la valoración sociocultural participativa de los SE, ya que incorporamos los diversos valores socioculturales de un ecosistema costero, e identificamos los elementos ecosistémicos relevantes y aquellos que necesitan de mayor sensibilización entre las comunidades locales. Estos SE son específicos del contexto por lo que pueden ser integrados en la toma de 
decisiones local y facilitar el comportamiento a favor de la protección del humedal.

Finalmente, el conocimiento de la dependencia, en gran parte, de los recursos que brinda el humedal y la importancia sociocultural del ecosistema, revela que mantener los diferentes SE, es equivalente a mantener el bienestar de las comunidades locales; y debe ser considerado antes de asumir cualquier estrategia para proteger el humedal costero Laguna de Nuxco.

SANDY A. MEDINA-VALDIVIA. Doctorante en Ciencias Ambientales en el Centro de Ciencias de Desarrollo Regional, Universidad Autónoma de Guerrero. Bióloga y maestra en Ciencias por la Universidad Autónoma de Guerrero, con especialidad en Desarrollo Sustentable del Turismo. Colabora con equipos interdisciplinarios y cuenta con amplia experiencia en el desarrollo de proyectos ambientales tales como estudios de impacto ambiental, estudios de biodiversidad, conservación de la naturaleza y monitoreo comunitario. Ha realizado estancias académicas en la Universidad de Quintana Roo, en México; Universidad de Luxemburgo, en Luxemburgo; y Universidad de Granada en España. Email: sandyastridmedina@ gmail.com

CARMEN MAGANDA-RAMíREZ. Investigador Titular "A" en la Red de Ambiente y Sustentabilidad del Instituto de Ecología A.C. Investigador Nacional Nivel 1 (SNI). Doctora en Antropología por el Centro de Investigaciones y Estudios Superiores en Antropología Social (CIESAS). Cuenta con tres posdoctorados por University of California, San Diego, CA; Université de Lille 1, Francia; y Laboratoire de Sciences Politiques, Universit' du Luxembourg. Sus principales líneas de investigación son, el manejo de recursos naturales y estratégicos, desarrollo sustentable, relación sociedad-naturaleza, aguas transfronterizas y seguridad ambiental con enfoque de la ecología política; asimismo, cuenta con publicaciones destacadas en temas relacionados con las Ciencias de la Sostenibilidad. Email: carmen.maganda@inecol.mx

R. CARLOS ALMAZÁN-NÚÑEZ. Profesor-investigador adscrito a la Facultad de Ciencias Químico-Biológicas de la UAGro. Doctor en Ciencias Biológicas por la Universidad Autónoma Metropolitana. Sus principales líneas de investigación son: la ecología de poblaciones y comunidades; el modelado de nichos ecológicos; la ecología de interacciones bióticas; y la biología de la conservación. Es miembro del Sistema Nacional de Investigadores nivel 1. Ha publicado 39 artículos científicos en revistas JCR y del Conacyt y cuenta con 3 capítulos de libro. Ha dirigido 27 tesis de licenciatura y 7 de maestría, y actualmente dirige 5 tesis de maestría y 2 de doctorado. Email: oikos79@yahoo.com.mx

AMÉRICA L. RODRÍGUEZ-HERRERA. Docente-investigadora del Doctorado en Ciencias Ambientales, Universidad Autónoma de Guerrero. Doctora en Antropología Social. Es miembro del Sistema Nacional de Investigadores nivel 1. Participa en el Cuerpo Académico consolidado Gestión y Ambiente, en ámbitos: experien- 
cias participativas para la gestión y los saberes ambientales. Imparte cursos en el área Epistemología, Pensamiento Sistémico, Métodos Cualitativos y Perspectiva Socioambiental en la gestión de recursos naturales. Ha publicado sobre tecnología tradicional en irrigación de la Montaña de Guerrero, riesgo y contaminación en la cuenca del río La Sabana; Ha dirigido tesis de doctorado, maestría y Licenciatura. Email: amerodriguez@gmail.com

COLUMBA RODRÍGUEZ-ALVISO. Docente-investigadora del Doctorado en Ciencias Ambientales de la Universidad Autónoma de Guerrero. Doctora en Geografía (UNAM). Tiene una investigación posdoctoral en Ciencias Humanas (Universidad del Zulia, Venezuela). Es líder del Cuerpo Académico Innovación Educativa y Sustentabilidad (consolidado); trabaja los temas: educación ambiental para la sustentabilidad y geografía ambiental. Dirigió 4 tesis de doctorado y actualmente dirige 4; ha participado como asesora en 16 tesis de licenciatura, maestría y doctorado. Ha realizado estancias académicas en universidades de México, Colombia y España. Ha participado en 23 artículos; nueve en catálogos JCR, Scopus y Conacyt; también en cuatro capítulos de libro. Ha participado en congresos nacionales e internacionales en México, España, Colombia y Ecuador. Email: columba26@ yahoo.com

JOSÉ LUIS ROSAS-ACEVEDO Docente-investigador del Doctorado en Ciencias Ambientales de la Universidad Autónoma de Guerrero. Biólogo, con Maestría en Entomología y Acarología. Doctorado en Biotecnología. Jefe del Laboratorio de Biomonitoreo y Control Biológico. 35 años de experiencia en docencia e investigación. Pertenece al CA-UAGro-29. Ambiente y Desarrollo (Consolidado). Líneas de investigación: Evaluación y manejo sustentable de los recursos naturales y, Saberes Ambientales y educación para el desarrollo sustentable. Estudios recientes sobre Macroinvertebrados acuáticos (Bioindicadores de calidad del agua) y Patología de insectos (Hongos entomopatógenos). Email: jlrosas71@yahoo.com

\section{REFERENCIAS}

Arias-Arévalo, P., Martín-López, B., \& Gómez-Baggethun, E. (2017). Exploring intrinsic, instrumental, and relational values for sustainable management of social-ecological systems. Ecology and Society 22(4), 43. https://doi.org/10.5751/ ES-09812-220443.

Boeraeve, F., Dufrene, M., De Vreese, R., Jacobs, S., Pipart, N., Turkelboom, F., Verheyden, W., \& Dendoncker, N. (2018). Participatory identification and selection of ecosystem services: Building on field experiences. Ecology and Society 23(2), 27. https://doi.org/10.5751/ES-10087-230227.

Burdon, D., Potts, T., McKinley, E., Lew, S., Shilland, R., Gormley, K., Thomson, S., \& Forster, R. (2019). Expanding the role of participatory mapping to assess ecosystem service provision in local coastal environments. Ecosystem Services 39(October), 101009. https://doi.org/10.1016/j.ecoser.2019.101009. 
Chan, K. M. A., Balvanera, P., Benessaiah, K., Chapman, M., Díaz, S., Gómez-Baggethun, E., Gould, R., Hannahs, N., Jax, K., Klain, S., Luck, G. W., MartínLópez, B., Muraca, B., Norton, B., Ott, K., Pascual, U., Satterfield, T., Tadaki, M., Taggart, J., \& Turner, N. (2016). Why protect nature? Rethinking values and the environment. Proceedings of the National Academy of Sciences of the United States of America 113(6), 1462-1465. https://doi.org/10.1073/pnas.1525002113.

Consejo Nacional de Población, CONAPO (2015). Índice de marginación por entidad federativa y municipio 2015. http://www.conapo.gob.mx/es/CONAPO/ Indices_de_Marginacion.

De Groot, D., Brander, L., \& Max Finlayson, C. (2018). Wetland ecosystem services. En C.M. Finlayson, M. Everard, K. Irvine, R. McInnes, B. Middleton, A. van Dam, N.C. Davidson (Eds.), The Wetland Book: I: Structure and Function, Management, and Methods (pp. 323-333). New York: Springer. https://doi .org/10.1007/978-90-481-9659-3_66.

Díaz, S., Pascual, U., Stenseke, M., Martín-López, B., Watson, R. T., Molnár, Z., Hill, R., Chan, K. M. A., Baste, I. A., Brauman, K. A., Polasky, S., Church, A., Lonsdale, M., Larigauderie, A., Leadley, P. W., Van Oudenhoven, A. P. E., Van Der Plaat, F., Schröter, M., Lavorel, S., \& Shirayama, Y. (2018). Assessing nature's contributions to people: Recognizing culture, and diverse sources of knowledge, can improve assessments. Science 359(6373), 270-272. https://doi .org/10.1126/science.aap8826.

Hernández-Félix, L., Molina-Rosales, D., \& Agraz-Hernández, C. (2017). Servicios ecosistémicos y estrategias de conservación en el manglar de isla arena. Agricultura, Sociedad y Desarrollo 14(3), 427-449. ISSN: 1870-5472.

Jacobs, S., Dendoncker, N., Martín-López, B., Barton, D. N., Gómez-Baggethun, E., Boeraeve, F., McGrath, F. L., Vierikko, K., Geneletti, D., Sevecke, K. J., Pipart, N., Primmer, E., Mederly, P., Schmidt, S., Aragão, A., Baral, H., Bark, R. H., Briceno, T., Brogna, D., . . Washbourn, C. L. (2016). A new valuation school: Integrating diverse values of nature in resource and land use decisions. Ecosystem Services 22(December), 213-220. https://doi.org/10.1016/j.ecoser.2016.11.007.

Langholz, J., Sand, K., Raak, L., Berner, A., Anderson, H., Geels, B., McKeehan, A., \& Nelsen, A. (2013). Strategies and tactics for managing environmental conflicts: Insights from Goldman Environmental Prize recipients. Journal of Natural Resources Policy Research 5(1): 1-17. https://doi.org/10.1080/19390459 .2013.771833.

Lara-Pulido, J. A., Guevara-Sanginés, A., \& Arias Martelo, C. (2018). A metaanalysis of economic valuation of ecosystem services in Mexico. Ecosystem Services 31(June), 126-141. https://doi.org/10.1016/j.ecoser.2018.02.018.

Maass, M. (2017). Integrating food-water-energy research through a socioecosystem approach. Frontiers in Environmental Science 5(August), 1-8. https:// doi.org/10.3389/fenvs.2017.00048.

Marín-Muñiz, J. L., Hernández Alarcón, María E., Rivera, Evodia Silva, \& MorenoCasasola, P. (2016). Percepciones sobre servicios ambientales y pérdida de humedales arbóreos en la comunidad de Monte Gordo, Veracruz. Madera y bosques 22(1), 53-69. 
Martín-López, B., Gómez-Baggethun, E., García-Llorente, M., \& Montes, C. (2014). Trade-offs across value-domains in ecosystem services assessment. Ecological Indicators 37(February): 220-228. https://doi.org/10.1016/j.ecolind.2013.03.003.

Medina-Valdivia, S. (2019). Pescadores y pescadoras, una forma de vida alrededor de los humedales. Regions and Cohesion 9(2), 113-116. https://doi.org/10.3167/ reco.2019.090206.

Medina-Valdivia, S. A., \& Maganda-Ramírez, M. (2019). ¿Es posible restaurar humedales mientras se promueve el desarrollo comunitario? Un reto transdisciplinario en la subcuenca Nuxco. En D. Barkin \& G. Carrillo, Ecological economics and socio-ecologicals movements: Science, policy and challenges to global processes in a troubled world (pp. 231-245). México.

Millennium Ecosystem Assessment-MEA. (2005). Capital natural y bienestar humano 1-28. Retrieved from https://www.millenniumassessment.org.

Pascual, U., Balvanera, P., Díaz, S., Pataki, G., Roth, E., Stenseke, M., Watson, R. T., Başak Dessane, E., Islar, M., Kelemen, E., Maris, V., Quaas, M., Subramanian, S. M., Wittmer, H., Adlan, A., Ahn, S. E., Al-Hafedh, Y. S., Amankwah, E., Asah, S. T., . . . Yagi, N. (2017). Valuing nature's contributions to people: The IPBES approach. Current Opinion in Environmental Sustainability 26, 7-16. https://doi.org/10.1016/j.cosust.2016.12.006.

Perevochtchikova, M., De la Mora-De la Mora, G., Hernández Flores, J. Á., Marín, W., Langle Flores, A., Ramos Bueno, A., \& Rojo Negrete, I. A. (2019). Systematic review of integrated studies on functional and thematic ecosystem services in Latin America, 1992-2017. Ecosystem Services 36(April), 100900. https://doi.org/10.1016/j.ecoser.2019.100900.

Rey-Valette, H., Mathé, S., \& Salles, J. M. (2017). An assessment method of ecosystem services based on stakeholders perceptions: The Rapid Ecosystem Services Participatory Appraisal (RESPA). Ecosystem Services 28(December), 311-319. https://doi.org/10.1016/j.ecoser.2017.08.002.

Rincón-Ruiz, A., Arias-Arévalo, P., Núñez Hernández, J. M., Cotler, H., Aguado Caso, M., Meli, P., Tauro, A., Ávila Akerberg, V. D., Avila-Foucat, V. S., Cardenas, J. P., Castillo Hernández, L. A., Castro, L. G., Cerón Hernández, V. A., Contreras Araque, A., Deschamps-Lomeli, J., Galeana-Pizaña, J. M., Guillén Oñate, K., Hernández Aguilar, J. A., Jimenez, A. D., . . Waldron, T. (2019). Applying integrated valuation of ecosystem services in Latin America: Insights from 21 case studies. Ecosystem Services 36(April), 100901. https://doi .org/10.1016/J.ECOSER.2019.100901.

Scholte, S. S. K., van Teeffelen, A. J. A., \& Verburg, P. H. (2015). Integrating socio-cultural perspectives into ecosystem service valuation: A review of concepts and methods. Ecological Economics 114(June), 67-78. https://doi.org/ 10.1016/j.ecolecon.2015.03.007.

Souza-Queiroz, L., Rossi, S., Calvet-Mir, L., Ruiz-Mallén, I., García-Betorz, S., Salvà-Prat, J., \& de Andrade Meireles, A. J. (2017). Neglected ecosystem services: Highlighting the socio-cultural perception of mangroves in decision-making processes. Ecosystem Services 26(August), 137-145. https://doi .org/10.1016/j.ecoser.2017.06.013. 
Turton, A. (2020). Barriers and borders. Regions and Cohesion 10(2), 87-98. https:// doi.org/10.3167/reco.2020.100206.

Valderrama Landeros, L. H., Rodríguez Zúñiga, M. T., Troche-Souza, Carlos Velázquez Salazar, Samuel Villeda Chávez, E., Alcántara Maya, J. A., \& Vázquez Balderas, Berenice Cruz López, \& María Isabel Ressl, R. (2017). Manglares de México: actualización y exploración de los datos del sistema de monitoreo 1970/1980-2015. Ciudad de México: Comisión Nacional para el Conocimiento y Uso de la Biodiversidad.

Vences-Martínez, J. Á., Sampedro Rosas, M. L., Castillo Elías, B., Olmos Martínez, E., Juárez López, A. L., \& Reyes Umaña, M. (2016). Afectación del manglar por actividades antropogénicas en la subcuenca de Nuxco, Guerrero, México. Revista Mexicana de Agroecosistemas 3(2), 163-174.

Vences-Martínez, J. Á., Sampedro Rosas, M. L., Castillo Elías, B., Olmos Martínez, E., Rosas Guerrero, V. M., Juárez López, A. L., \& Reyes Umaña, M. (2016). Perception and environmental knowledge of anthropogenic activities in a coastal community. International Journal of Current Research 10(3), 66750-66755.

Vidal, L., Vallarino, A., Benítez, I., \& Correa, J. (2015). Implementación del plan estratégico Ramsar en humedales costeros de la Península de Yucatán: normativas y regulación. Implementation of the Ramsar Strategic Plan in Coastal Wetlands of the Península de Yucatán: Regulations and Normativity 43(5), 873-887. Descargado de https://www.redalyc.org/articulo.oa?id=1750/175042668007.

Villaseñor, E., Porter-Bolland, L., Escobar-Sarria, F., et al. (2020). Selection of indicators as a tool for negotiating objectives and evaluating targets within participatory monitoring. Sustainability Science 15, 1051-1065. https://doi .org/10.1007/s11625-020-00795-w.

\section{Participative assessment of ecosystem services in Laguna de Nuxco, Guerrero}

Sandy A. Medina-Valdivia, Carmen Maganda-Ramírez, R. Carlos

Almazán-Núñez, América L. Rodríguez-Herrera, Columba Rodríguez-Alviso, José Luis Rosas-Acevedo

Abstract: How do societies value and interact with ecosystem services
(ES) to favor their long-term conservation? Under the premise that socio-
cultural assessment of ES offers an expanded perspective for potential
societal nature contributions, we present here the empirical results of a
participative assessment of ES in the Laguna de Nuxco coastal wetland
in 2019 . The methodological design includes the combination of applied
qualitative tools on site and quantitative methods for analysis of infor-
mation. The results emphasize that the ES of the wetland support socio-
economic needs and important socio-cultural community traits, such as
cultural heritage and identity as fishing communities. We identify emerg- 
ing socio-ecological conflicts that promote the decrease of ES. We suggest conceptual and methodological adaptations for the participative assessment of ES on the local scale.

Keywords: coastal wetlands, Guerrero communities, participative tools, socio-cultural perception, socio-ecosystems

\section{Évaluation participative des services écosystémiques à Laguna de Nuxco, au Guerrero}

\section{Sandy A. Medina-Valdivia, Carmen Maganda-Ramírez, R. Carlos}

Almazán-Núñez, América L. Rodríguez-Herrera, Columba Rodríguez-Alviso, José Luis Rosas-Acevedo.

Résumé: Comment les sociétés valorisent-elles et interagissent-elles avec les services écosystémiques (SE) pour favoriser leur conservation à long terme? Le principe de l'évaluation socioculturelle des SE offre une perspective élargie des contributions potentielles société-nature, et est utilisé pour présenter les résultats empiriques d'une évaluation participative des SE réalisée en 2019 dans la zone humide côtière de Laguna de Nuxco, au Mexique. La méthodologie comprenait la combinaison d'outils qualitatifs sur site et d'outils quantitatifs pour l'analyse de l'information. Les résultats soulignent que les SE de la zone humide répondent aux besoins socio-économiques et à des aspects socioculturels tels que le patrimoine culturel et l'identité en tant que communautés de pêcheurs. Nous identifions les conflits socio-écologiques émergents qui favorisent la diminution des SE. Nous suggérons des adaptations conceptuelles et méthodologiques pour l'évaluation participative des SE à l'échelle locale.

Mots clés: communautés Guerrero, outils participatifs, perception socioculturelle, socio-écosystèmes, zones humides côtières. 